\title{
The risk of hospitalization for respiratory tract infection (RTI) in children who are treated with high-dose IVIG in Kawasaki Disease: a nationwide population-based matched cohort study
}

\author{
Wei-Te Lei ${ }^{1}$, Chien-Yu Lin ${ }^{1,2}$, Yu-Hsuan Kao ${ }^{3}{ }^{\text {, Cheng-Hung Lee }}{ }^{4}$, Chao-Hsu Lin ${ }^{1}$, Shyh-Dar Shyur ${ }^{3}$, \\ Kuender-Der Yang ${ }^{3}$, Jian-Han Chen ${ }^{\text {Corresp. } 5}$ \\ 1 Department of Pediatrics, Hsinchu MacKay Memorial Hospital, Hsinchu, Taiwan \\ 2 College of Medicine and Veterinary Medicine, the University of Edinburgh, Scotland, UK \\ 3 Department of Pediatrics, MacKay Children's Hospital, Taipei, Taiwan \\ 4 Department of General Surgery, Buddhist Dalin Tzu Chi Hospital, Chia-Yi, Taiwan \\ 5 Department of General Surgery, E-Da Hospital, Kaohsiung, Taiwan \\ Corresponding Author: Jian-Han Chen \\ Email address: jamihan1981@gmail.com
}

Background: Kawasaki disease (KD) is an immune-mediated systemic vasculitis and infection plays an important role in the pathophysiology of KD. The susceptibility to infectious disease in patients with KD remains largely unclear. This study aimed to investigate the risk of respiratory tract infection (RTI)related hospitalizations in children with KD.

Methods: Data from Taiwanese National Health Insurance Research Database was analyzed. We excluded patients with history of congenital abnormality, allergic diseases, or hospitalization history. Children with KD were selected as KD group and age- and sex-matched non-KD patients were selected as control group with 1:4 ratio. Both cohorts were tracked for 1 year to investigate the incidences of RTIrelated hospitalizations. Cox regression hazard model was used to adjust for confounding factors and calculate the adjusted hazard ratio (aHR).

Results: Between January 1996 and December 2012, 4,973 patients with KD were identified as KD group and 19,683 patients were enrolled as control group. An obviously reduced risk of RTI-related hospitalizations was observed in KD patients (aHR: 0.75, 95\% confidence interval: 0.66-0.85). The decreased risk persisted through the first 6-months follow-up period with a peak protection in 3-6 months (aHR: 0.49, 95\% confidence interval: 0.37-0.64).

Conclusions: KD patients had approximately half reduction of risk for RTI-related hospitalizations. The protective effects persisted for at least 6 months. Further studies are warranted to elucidate the entire mechanism and investigate the influences of intravenous immunoglobulin. 


\section{Research Article}

\section{The risk of hospitalization for respiratory tract infection (RTI) in}

\section{3 children who are treated with high-dose IVIG in Kawasaki Disease: a}

\section{4 nationwide population-based matched cohort study}

5 Short title: Respiratory tract infection in Kawasaki disease

6 Type of article: Original article

7 Wei-Te Lei ${ }^{1}$, Chien-Yu Lin ${ }^{1,2}$, Yu-Hsuan Kao ${ }^{3}$, Cheng-Hung Lee ${ }^{4}$, Chao-Hsu Lin ${ }^{1}$, Shyh-Dar Shyur ${ }^{3}$,

8 Kuender-Der Yang ${ }^{3}$, Jian-Han Chen ${ }^{5, *}$

$9{ }^{1}$ Department of Pediatrics, Hsinchu MacKay Memorial Hospital, Hsinchu, Taiwan

$10{ }^{2}$ College of Medicine and Veterinary Medicine, the University of Edinburgh, Scotland, UK

$11{ }^{3}$ Department of Pediatrics, MacKay Children's Hospital, Taipei, Taiwan

$12{ }^{4}$ Department of General Surgery, Buddhist Dalin Tzu Chi Hospital, Chia-Yi, Taiwan

$13{ }^{5}$ Department of General Surgery, E-Da Hospital, Kaohsiung, Taiwan School of Medicine, I-sou

14 University, Kaohsiung, Taiwan

17 Address correspondence to: Dr. Jian-Han Chen, MD

Dr. Jian-Han Chen,

19 Department of General Surgery, E-Da Hospital, Kaohsiung, Taiwan School of Medicine, I-sou

20 University, Kaohsiung, Taiwan, No.1, Yida Rd., Yanchao Dist., Kaohsiung City 824, Taiwan.

21 Tel: +886-7-615-0011 
22 Fax: +822-7-615-5157

\section{Email: jamihan1981@gmail.com}

24

25

26 


\section{Original Study}

28 The risk of hospitalization for respiratory tract infection (RTI) in

29 children who are treated with high-dose IVIG in Kawasaki Disease: a

30 nationwide population-based matched cohort study

32 Abstract

33 Background: Kawasaki disease (KD) is an immune-mediated systemic vasculitis and infection

34 plays an important role in the pathophysiology of KD. The susceptibility to infectious disease in

35 patients with KD remains largely unclear. This study aimed to investigate the risk of respiratory

36 tract infection (RTI)-related hospitalizations in children with KD.

37 Methods: Data from Taiwanese National Health Insurance Research Database was analyzed.

38 We excluded patients with history of congenital abnormality, allergic diseases, or

39 hospitalization history. Children with KD were further selected as KD group and age- and sex-

40 matched non-KD patients were selected as control group with 1:4 ratio. Both cohorts were

41 tracked for 1 year to investigate the incidences of RTI-related hospitalizations. Cox regression

42 hazard model was used to adjust for confounding factors and calculate the adjusted hazard

43 ratio (aHR).

44 Results: Between January 1996 and December 2012, 4,973 patients with KD were identified as

$45 \mathrm{KD}$ group and 19,683 patients were enrolled as control group. An obviously reduced risk of RTI-

46 related hospitalizations was observed in KD patients (aHR: 0.75 , 95\% confidence interval: 0.66 - 
47 0.85). The decreased risk persisted through the first 6-months follow-up period with a peak

48 protection in 3-6 months (aHR: $0.49,95 \%$ confidence interval: 0.37-0.64).

49 Conclusions: KD patients had approximately half reduction of risk for RTI-related

50 hospitalizations. The protective effects persisted for at least 6 months. Further studies are

51 warranted to elucidate the entire mechanism and investigate the influences of intravenous

52 immunoglobulin. 


\section{INTRODUCTION}

56 Kawasaki disease (KD) is an important cause of systemic vasculitis and the main cause of

57 heart disease in childhood.(Newburger et al. 2004) Although the etiology is not well known,

58 specific infection agents may cause genetic susceptibility in certain children.(Wang et al. 2005;

59 Weng et al. 2017) Globally, Taiwan has the third highest incidence of KD, second only to Korea

60 and Japan.(Huang et al. 2009) An aberrant immune response is also believed to be key in the

61 preclinical and acute stages of this disease.(Hara et al. 2016; Matsubara et al. 2005) Treatment

62 with high-dose intravenous immunoglobulin (IVIG) along with moderate to high doses of aspirin

63 is the current gold standard.(Newburger et al. 2004) In Taiwan, a high dose $2 \mathrm{~g} / \mathrm{kg} / \mathrm{dose}$ IVIG is

64 the standard treatment of KD. While the mechanisms underlying the beneficial effects of IVIG

65 remain unknown, its broad anti-inflammatory effect is believed to be an important factor.

66 Coronary artery aneurysm is the main concern of KD but children with KD also have higher risk

67 of some systemic diseases, such as atopic dermatitis.(Abrams et al. ; Wei et al. 2014) Clinically,

68 no obvious risks of infectious diseases are observed in patients with KD and children with KD

69 are not regarded as immunocompromised patients. However, studies investigating the

70 infectious risk are limited and the susceptibility to infectious disease in patients with KD

71 remains largely unclear.

Human products of IVIG is made by the pooled human plasma of approximately a

thousand blood donors.(Hemming 2001; Perez et al. 2017) Initially, it was intended to protect

patients with primary immunodeficiency (PID) against infection. The ability of anti-inflammation

and immune-modulation of IVIG has been recognized and its use is increasing in a variety of

77 diseases.(Siberil et al. 2007; Wong \& White 2016) The US Food and Drug Administration has 
with hypogammaglobulinemia and recurrent bacterial infection due to B-cell chronic lymphocytic leukemia; prevention of pneumonitis and graft-versus-host disease following bone marrow transplantation; reduction of severe infection in HIV-infected children; prevention of coronary artery aneurysms in KD; and decrease bleeding in idiopathic thrombocytopenic purpura (ITP).(Perez et al. 2017) IVIG is composed by "protective" immunoglobulin and the recommendation of IVIG use in treatment or prophylaxis in patients with various kinds of immunodeficiency is well documented.(Ammann et al. 1982; Hemming 2001; Keller \& Stiehm 2000; Malik et al. 1991; Orange et al. 2010) However, the protective effects of IVIG against infection in other patients remain largely unclear. Preterm babies have relatively lower level of immunoglobulins and are prone to infection. Studies investigating the prophylactic effects of IVIG in preterm patients demonstrated a $3 \%$ reduction in sepsis and $4 \%$ reduction in any serious infection. But IVIG use was not associated with clinical outcomes.(Ohlsson \& Lacy 2013) Further studies are required to elucidate the protective effects of IVIG.

3 In our daily practice, we found children with KD seemed to have less severe infection after treatment of KD but no related studies were found in the literature. KD is an immune-mediated disease and patients with KD may have different susceptibility to infectious disease compared with general population. Furthermore, KD is the major indication for IVIG use and the protective effects of IVIG against infection may contribute to the observed decreased risk.(Perez et al. 2017) Therefore, a nationwide population-based study was conducted to investigate the incidences of hospitalization for respiratory tract infections (RTI) in children with KD and explore the potentially protective effects of IVIG. 
103 MATERIALS AND METHODS

\section{Data sources}

105 Data were retrieved from the National Health Insurance Research Database (NHIRD) of the

106 National Health Research Institutes. The Taiwan's National Health Insurance (NHI) is a

107 nationwide program implemented in March 1995. Approximately 99\% of 23.74 million

108 residents in Taiwan have been enrolled in this program.(Liu et al. 2017) The database contains

109 universal files and longitudinal medical records from 1996 to 2013, such as demographic

110 characteristics, inpatient and outpatient data, dates of admission, diagnostic codes, and

111 medical prescriptions. All diseases are coded with the International Classification of Disease,

112 Ninth Revision, Clinical Modification (ICD-9-CM).(American Hospital Association et al. 1990) This

113 study was reviewed and approved by the Institutional Review Board of Buddhist Tzu Chi

114 Hospital, Dalin, Taiwan (IRB approval number: B10503021). The institutional review board

115 exempted consent requirement.

116

117 Study design and population

118 Two cohorts involved in our study were chosen from the NHIRD: KD group and control

119 group. Figure 1 describes the cohort study framework. We extracted patient data regarding

120 inpatient expenditures by admissions from the NHIRD database between January 1, 1996 and

121 December 31, 2013. In order to minimize the potential bias of selection, patients with history of

122 congenital abnormality, allergic diseases, or hospitalization history were excluded in both

123 cohorts. Patients with KD were selected as KD group and the exclusion criteria included (1)

124 recurrent KD, (2) birth before 1996, and (3) age >= 6 years old. (4) with congenital abnormality

125 and prenatal disease, (5) with history of asthma, atopic dermatitis and allergic rhinitis, (6) with

126 respiratory tract infection history. Meanwhile, patients without KD were selected as control 
127 group and the exclusion criteria comprised (1) age $\geqq 6$ years old, ( 2 ) with any hospitalization

128 history including respiratory tract infections, congenital abnormality and prenatal disease, and

129 (4) with history of asthma, atopic dermatitis and allergic rhinitis. Patients were randomly

130 selected from non-KD cohort by 1:4 propensity score match with parameters including age and

131 sex with patients from KD cohort. We followed both cohorts for 1 year to investigate the

132 incidences of RTI-related hospitalizations. In this cohort study, we identified 17,580 patients

133 who were admitted with a diagnostic code of KD (ICD-9, 446.1). Patients who were born before

134 January 1, $1996(n=2,007)$ and those who were admitted after December 31, $2012(n=955)$

135 were excluded. In addition, 2,497 patients with recurrent KD, 354 patients aged >= 6 years, 16

136 patients with undetermined sex, 2,977 patients with congenital abnormalities and prenatal

137 disease, 190 patients with past history of asthma, atopic dermatitis and allergic rhinitis, and

1383,611 patients with history of respiratory tract infections were also excluded. Finally, 4,973

139 patients aged $<6$ years and newly diagnosed with KD were included. The uses of IVIG were

140 validated by prescription records.

141

142 Study outcomes and follow-up duration

143 All patients were followed up for 1 year after enrollment. Censoring was defined as

144 withdrawal from the insurance program due to death, living abroad for $>6$ months, or missing

145 appointments for $>6$ months. The primary outcomes were to determine admissions for RTI-

146 related hospitalizations, including pneumonia, acute otitis media, acute bronchitis, influenza,

147 and acute tonsillitis. To identify accompanied respiratory tract infection, associated

148 comorbidities and congenital abnormalities, we searched diagnosis based on the categories of

149 Clinical Classification Software codes (CCS)(Supplement 1), which collapsed all ICD-9-CM's

150 diagnosis and procedure codes into clinically meaningful categories that are useful for 
151 presenting descriptive statics.(Thompson et al. 2006) The congenital abnormality (CCs-Multiple-

152 Diagnosis 14.x.x), prenatal disease (CCs-Multiple-Diagnosis 15.2-6), AOM (CCs-Multiple-

153 Diagnosis 6.8.1), pneumonia (CCs-Multiple-Diagnosis 8.1.1), tonsillitis (CCs-Multiple-Diagnosis

154 8.1.3), bronchitis (CCs-Multiple-Diagnosis 8.1.4), and asthma were identified. (CCs-Multiple-

155 Diagnosis 8.3.x). Other comorbidities including atopic dermatitis (ICD-9-CM codes 691.8) and

156 allergic rhinitis (ICD-9-CM codes 477.9) were also identified. The reason for choosing

157 pneumonia, AOM, bronchitis, and tonsillitis as the representation for RTI in this study is based

158 on the top ten diagnoses of children requiring emergency care and subsequent hospitalization

159 from a 10-year population-based nationwide analysis in Taiwan.(Jeng et al. 2014)

\section{Statistical analysis}

162 The incidence density rate of KD (per 1000 person-years) was measured based on national

163 live birth data. Differences in demographic characteristics, co-variables, and admissions for

164 respiratory infections between IVIG and control groups were analyzed using categorical

165 variables, the Student's $t$ test for continuous variables with normal contribution, and the

166 Mann-Whitney $U$ test for continuous variables without normal contribution. The Kaplan-Meier

167 methods with the log-rank test were used to compare the survival distributions between the

168 cohorts. $P$ values of $<0.2$ were inserted into Cox proportional hazards analysis to quantify the

169 risk of subsequent RTIs after adjusting for comorbidities. All statistical analyses were performed

170 with SPSS (Statistical Package for Social Science) statistical software version 22.0 (IBM, Armonk,

171 NY, USA). A 2-sided P value of $<0.05$ was considered statistically significant.

172 


\section{RESULTS}

174 We identified 4,973 patients as KD group and 19683 patients as non-KD group between

1751996 and 2013 in Taiwan. Table 1 demonstrated the baseline characteristics for the two

176 cohorts. Both cohorts showed a similar distribution of age; $73.80 \%$ and $73.88 \%$ patients were

177 aged $<2$ years in the KD and non-KD cohorts, and $26.20 \%$ and $26.12 \%$ patients were aged 2 to 6

178 years, in the KD and non-KD cohorts, respectively. The KD cohort had similar distribution of

179 gender with non-KD cohort.

180 The cumulative incidence of all RTI-related hospitalizations was significantly lower in the

181 KD cohort ( $p<0.001$, log-rank test) than in the non-KD cohort (Figure 2). Furthermore, the

182 cumulative incidence of hospitalizations due to pneumonia, AOM, and acute bronchiolitis were

183 all lower in the KD cohort $(p=0.010, p=0.010, p=0.033$, respectively) than in the non-KD

184 cohort (Figures $\mathbf{3}, \mathbf{4}, \mathbf{5}$ ). There was no significant difference in the cumulative incidence of

185 hospitalization due to tonsillitis between both group $(p=0.819)$.

The hazard ratios of RTI-related hospitalizations were listed in Table 2 . The overall

incidence ratio of all RTI-related hospitalizations was 0.87 -fold lower in the KD cohort [16.72 vs

19.18 per 1000 person-months, 95\% confidence interval (Cl): 0.76-0.99; $p<0.05]$. Moreover, the incidence ratio of pneumonia-related hospitalizations was also lower in the KD cohort

191 (15.79 vs 20.52 per 1000 person-months, 95\% Cl: 0.61-0.96; $p<0.05)$. After adjusting for

192 potential confounders, adjusted hazard ratios (aHRs) were lower in the KD cohort for almost all

193 RTI-related hospitalizations (aHR: 0.75; 95\% Cl: 0.66-0.85; $p<0.001$ ), pneumonia-related

194 hospitalizations (aHR: 0.64; 95\% Cl: 0.52-0.79; $P<0.001$ ), AOM-related hospitalizations (aHR:

$1950.61 ; 95 \% \mathrm{Cl}: 0.42-0.90 ; p<0.05)$, acute bronchiolitis-related hospitalizations (aHR: 0.77; 95\%

$196 \mathrm{Cl}: 0.560-0.99 ; p=0.042$ ), and tonsillitis (aHR: $1.04 ; 95 \% \mathrm{Cl}: 0.76-1.41 ; p=0.845)$. The reduction 
197 of RTI-related hospitalizations was observed in both girls and boys. Girls in the KD cohort

198 displayed a 0.75-fold decreased incidence of RTI-related hospitalizations compared with girls in

199 the control cohort (girls: aHR, 0.75; 95\% Cl: 0.60-0.93; $P<0.05$; boys: aHR, $0.74 ; 95 \% \mathrm{Cl}$ : $0.63-$

200 087.; $p<0.001)$. Moreover, the incidence of RTI-related hospitalizations increased with age in

201 the KD cohort whereas decreased with age in non-KD cohort. The highest incidence of RTI-

202 related hospitalizations was seen in non-KD group patients aged 0-2 years (19.34 per 1000

203 person-months). The risk for RTI-related hospitalizations was lower in the KD group than in the

204 non-KD cohort for patients aged $0-2$ years: aHR, $0.69 ; 95 \% \mathrm{Cl}: 0.59-0.80 ; P<0.001)$.

205 We further analyzed the aHR of RTI-related hospitalizations within the 12-month follow-up 206 period to investigate the affecting duration of IVIG. We further stratified the episodes of

207 hospitalizations by follow-up time into four periods (Table 3). The incidence of RTI-related

208 hospitalizations in both cohorts decreased as the follow-up time increased in the first 6 months.

209 In these follow-up periods, the risk of RTI-related hospitalizations was lower in the KD cohort

210 than in the control cohort and the lowest aHR was observed in the 3-6-month follow-up group

211 (aHR: 0.49; 95\% Cl: 0.37-0.64; $p<0.001$ ).

212

213 


\section{Discussion}

215 Research evidence in the area of susceptibility to infection in patients with KD is scarce and

216 we found a decreased risk of subsequent hospitalizations due to pneumonia, AOM, and acute

217 bronchiolitis in patients with KD within 6 months after discharge. We procured a marked lower

218 aHR of $0.75(95 \% \mathrm{Cl}: 0.66-0.85)$ for RTI-related hospitalizations in the KD cohort than in the

219 non-KD cohort. The protective effects persisted during the 6-month follow-up period with the

220 lowest aHR within the 3-6 months (aHR: $0.49,95 \% \mathrm{Cl}: 0.37-0.64$ ). Some protective effects from

221 RTI-related hospitalizations in the KD cohort seem to exist compared with the non-KD cohort.

222 Peak protective effects were within the first 3-6 months.

223

224 Although it has been frequently postulated that KD is caused by an aberrant immune

response after an infectious episode, the pathogenesis of KD is not fully understood. KD

patients are not regarded as immunocompromised individuals, alterations of immune systems

are believed to play important roles in KD.(Hara et al. 2016; Newburger et al. 2016; Wang et al.

2005) In the acute stage of KD, evidence has shown that the roles of T cell activation and

inflammatory cytokines are both critical.(Brogan et al. 2008; Lee et al. 2015) T cells such as Th1,

Th2, and Treg cells have all been identified to be involved in this stage. Cytokines derived from

231 Th1 cells (IL-2, IFN- $\gamma$, and IL-10) and Th2 cells (II-4 and IL-5) have also been identified to be

232 involved in the disease process.(Abe et al. 2005; Hsieh et al. 2011; Kimura et al. 2004;

233 Matsubara et al. 2005) T cell activation, along with cytokine-induced macrophage activation, is

234 critical to the pathogenesis of vascular endothelial damage. The subsequent infiltration of

neutrophils, plasma cells, and eosinophils in coronary arteries may cause the destruction of

236 arterial wall integrity and result in dilatation and aneurysm formation. Treg cells play an

237 important role in weakening the pathogenic effects of T cells in the destruction of coronary 
238 arteries.(Ye et al. 2016) Further studies have demonstrated significantly lower levels of Treg-

239 related gene expressions such as FOXP3, GITR, and CTLA4 in acute KD patients prior to

240 treatment compared with healthy controls.(Anthony \& Ravetch 2010; Ephrem et al. 2008) On

241 the other hand, the mechanism of immune regulation by IVIG in KD patients remains

242 unclear.(Burns \& Franco 2015; Ephrem et al. 2005; Perez et al. 2017; Siberil et al. 2007) Many

243 potential mechanisms have been proposed, such as agent-specific neutralizing antibodies,

244 decreased proliferation of Th17 cells, and reduced cytokine release. IVIG stimulates an

245 immature myeloid population of IL-10-secreted DCs, which leads to the expansion of induced

246 Treg cells. Treg cells then recognize the Fc of IgG and block the activated Fcy receptor and

247 stimulate the inhibitory Fcy RIIb receptor. In addition, IVIG contains various antibodies specific

248 to a vast range of pathogens and it may be taken for granted that IVIG protects the KD group

249 from subsequent RTI-related hospitalizations. However, immunoinflammatory responses are

250 complex and both KD and IVIG may contribute to the reduction of RTI-related hospitalizations.

251 Further studies are warranted to clarify the entire mechanisms of the observed protective

252 effects in present study.

253

254 Prophylactic IVIG use in immunocompromised patients has been well documented but

255 studies investigating IVIG use in non-immunocompromised patients are limited.(Keller \& Stiehm

256 2000; Mouthon \& Lortholary 2003; Orange et al. 2010; Perez et al. 2017) We found an obvious

257 reduction of risk for RTI-related hospitalizations in IVIG-treated patients. However, in addition

258 to flu-like symptoms, severe adverse effects may occur after IVIG use, such as anaphylaxis and

259 thrombo-embolism.(Hefer \& Jaloudi 2004; Milani et al. 2009) Regularly prophylactic IVIG use in

260 immunocompetent patients is not suggested. For high risk groups, costs and benefits should be 
261 evaluated carefully to make a decision of IVIG use.(Ohlsson \& Lacy 2013; Perez et al. 2017) In

262 the present study, no severe adverse effects were noted after IVIG use.

263

264 For patients with PID, monthly IVIG supplement is suggested.(Hemming 2001; Keller \&

265 Stiehm 2000; Wong \& White 2016) Waning of immunoglobulin is a main concern of IVIG

266 treatment. Half-lives of immunoglobulins vary from 2.5 to 23 days in previous reports.(Bonilla

267 2008; Leuridan et al. 2010) The required serum IgG level for adequate protection and the

268 achieved concentration after IVIG supplement are different in different IVIG products and

269 dosages.(Orange et al. 2010; Perez et al. 2017) Our study showed the protective effects could

270 persist up to 6 months after IVIG administration, with a peak in 3-6 months. For patients with

$271 \mathrm{KD}$, high dose of IVIG treatment $(2 \mathrm{~g} / \mathrm{kg} /$ dose $)$ is the standard treatment and higher dose may

272 contribute to longer protection. Furthermore, we are curious how long will the protective

273 effects exist. Within the current 1-year follow-up period, the aHR of RTI-related hospitalizations

274 decreased in the KD group in 0-3 months and 3-6 months (Table 3, aHR: 0.68 and 0.49,

275 respectively) whereas the aHR of RTI-related hospitalization declared no difference between

276 the KD and non-KD group in 6-9 and 9-12 months. The abrupt disappear of decreasing aHR for

277 RTI-related hospitalization 6 months after KD may suggest that waning of IVIG-containing

278 antibodies occurred. It also provides a hint the influences of IVIG exist; differences in infectious

279 susceptibility of KD patients may not change by time. Moreover, it seems that patients in KD

280 cohort were less susceptible to pneumonia, AOM, and bronchiolitis but not tonsillitis. Therefore,

281 IVIG may have different protective effect on different type of respiratory tract infectious

282 disease. Longer follow-up periods may tell us the exact duration of protection on different

283 respiratory tract infectious disease and the baseline infectious susceptibility in both groups.

284 Further studies investigating the optimal dosage and interval of IVIG are also required. 
286 The strength of current study is a large population with standardized treatment. There

287 were several limitations to this study. First, the observed protective effects may attribute to 288 different susceptibility to infection in patients with KD itself or the immune regulatory effects of

289 IVIG. It's valuable to compare the infection risk in KD patients without IVIG but IVIG is the 290 standard treatment of KD. It's unethical to not use IVIG in patients with KD. Similarly, it's more

291 meaningful to compare the incidences of infection in general populations with and without IVIG.

292 However, IVIG is not commonly used in general population and it's difficult to recruit healthy

293 individuals to receive IVIG. Further studies are warranted to clarify the possible role of IVIG.

294 Second, although 1-year follow-up period is not short, longer follow-up time will provide more

295 information regarding the duration of protection. Third, RTI-infected patients treated as

296 outpatients with oral antibiotics were not included in our analysis. Although NHI in Taiwan

297 covered nearly all the populations and paid most of the hospitalization fees, some parents

298 chose to treat their children's illness such as pneumonia, AOM, and acute bronchiolitis at home.

299 Thus, the incidence of RTI-related hospitalization may have been underestimated for both

300 groups. The observed lower risk may indicate the less severe infection in patients with KD.

301 Furthermore, the NHIRD does not contain complete information such as laboratory data and

302 image reports. Hence, the causative pathogens of the RTIs remained unknown. The severity of

303 KD were not analyzed. Moreover, although the standard treatment of KD is IVIG administration

304 as $2 \mathrm{~g} / \mathrm{kg} /$ dose in Taiwan, individualized treatment may occur and a small part of KD patient may

305 receive different dosage of IVIG or no IVIG treatment. (Lin et al. 2015) The details of IVIG use

306 were not available and subgroup analyses were not performed. Finally, the current

307 retrospective cohort study could not clarify the underlying mechanism of the protective effects

308 from RTI-related hospitalizations in KD patients with IVIG treatment. Further investigation of 
309 these factors with a well-designed prospective cohort study with close monitoring of KD patient

310 serum markers in the convalescent stage is warranted.

311

\section{Conclusions}

313 In conclusion, this study found that children with KD had an approximately quarter

314 decreased risk of RTI-related hospitalization in the subsequent 1 year after IVIG treatment,

315 regardless of age, sex. This protective effect is not well understood but seemed to be well

316 maintained for at least for 6 months and then gradually decreased. Further researches are

317 warranted to illustrate the underpinning mechanisms and clarify the possible role of IVIG.

318

319

320

321

322

\section{Acknowledgments}

324 The authors would like to thank Dr. Hou-Ling Lung for providing the statistical opinion.

325

326 


\section{Figure legends}

328 Figure 1

329 Figure 1 demonstrate the flow chart of enrollment of study participants.

330 Figure 2

331 The Kaplan-Meier curve showed the accumulative incidences of all respiratory tract

332 infection-related hospitalizations between KD cohort and control cohort by time $(p<0.001)$.

\section{Figure 3}

334 The Kaplan-Meier curve showed the accumulative incidences of pneumonia-related

335 hospitalizations between KD cohort and control cohort by time $(p<0.010)$.

\section{Figure 4}

337 The Kaplan-Meier curve showed the accumulative incidences of AOM-related

338 hospitalizations between KD cohort and control cohort by time $(p<0.010)$.

\section{Figure 5}

340 The Kaplan-Meier curve showed the accumulative incidences of bronchiolitis-related

341 hospitalizations between KD cohort and control cohort by time $(p<0.033)$. 


\section{References}

345 Abe J, Jibiki T, Noma S, Nakajima T, Saito H, and Terai M. 2005. Gene expression profiling of the effect of high-dose intravenous Ig in patients with Kawasaki disease. J Immunol 174:5837-5845.

Abrams JY, Belay ED, Uehara R, Maddox RA, Schonberger LB, and Nakamura Y. Cardiac Complications, Earlier Treatment, and Initial Disease Severity in Kawasaki Disease. The Journal of Pediatrics 188:64-69. 10.1016/j.jpeds.2017.05.034

351

352

353

354

355

356

357

358

359

360

361

362

363

364

365

366

367

368

369

370

371

372

373

374

375

376

377

378

American Hospital Association, American Medical Record Association, Health Care Financing Administration, and Statistics NCfH. 1990. ICD-9-CM coding and reporting official guidelines. American Hospital Association, American Medical Record Association, Health Care Financing Administration, National Center for Health Statistics. J Am Med Rec Assoc 61:suppl 1-17.

Ammann AJ, Ashman RF, Buckley RH, Hardie WR, Krantmann HJ, Nelson J, Ochs H, Stiehm ER, Tiller T, Wara DW, and Wedgwood R. 1982. Use of intravenous $\gamma$-globulin in antibody immunodeficiency: Results of a multicenter controlled trial. Clinical Immunology and Immunopathology 22:60-67. http://dx.doi.org/10.1016/00901229(82)90022-8

Anthony RM, and Ravetch JV. 2010. A novel role for the IgG Fe glycan: the anti-inflammatory activity of sialylated IgG Fcs. J Clin Immunol 30 Suppl 1:S9-14. 10.1007/s10875-0109405-6

Bonilla FA. 2008. Pharmacokinetics of immunoglobulin administered via intravenous or subcutaneous routes. Immunol Allergy Clin North Am 28:803-819, ix. 10.1016/j.iac.2008.06.006

Brogan PA, Shah V, Clarke LA, Dillon MJ, and Klein N. 2008. T cell activation profiles in Kawasaki syndrome. Clin Exp Immunol 151:267-274. 10.1111/j.1365-2249.2007.03567.x

Burns JC, and Franco A. 2015. The immunomodulatory effects of intravenous immunoglobulin therapy in Kawasaki disease. Expert Rev Clin Immunol 11:819-825. 10.1586/1744666x.2015.1044980

Ephrem A, Chamat S, Miquel C, Fisson S, Mouthon L, Caligiuri G, Delignat S, Elluru S, Bayry J, Lacroix-Desmazes S, Cohen JL, Salomon BL, Kazatchkine MD, Kaveri SV, and Misra N. 2008. Expansion of CD4+CD25+ regulatory T cells by intravenous immunoglobulin: a critical factor in controlling experimental autoimmune encephalomyelitis. Blood 111:715-722. 10.1182/blood-2007-03-079947

Ephrem A, Misra N, Hassan G, Dasgupta S, Delignat S, Duong Van Huyen JP, Chamat S, Prost F, Lacroix-Desmazes S, Kavery SV, and Kazatchkine MD. 2005. Immunomodulation of 
379

380

381

382

383

384

385

386

387

388

389

390

391

392

393

394

395

396

397

398

399

400

401

402

403

404

405

406

407

408

409

410

411

412

413

414

autoimmune and inflammatory diseases with intravenous immunoglobulin. Clin Exp Med 5:135-140. 10.1007/s10238-005-0079-y

Hara T, Nakashima Y, Sakai Y, Nishio H, Motomura Y, and Yamasaki S. 2016. Kawasaki disease: a matter of innate immunity. Clin Exp Immunol 186:134-143. 10.1111/cei.12832

Hefer D, and Jaloudi M. 2004. Thromboembolic events as an emerging adverse effect during high-dose intravenous immunoglobulin therapy in elderly patients: a case report and discussion of the relevant literature. Annals of Hematology 83:661-665. 10.1007/s00277004-0895-2

Hemming VG. 2001. Use of Intravenous Immunoglobulins for Prophylaxis or Treatment of Infectious Diseases. Clinical and Diagnostic Laboratory Immunology 8:859-863. 10.1128/CDLI.8.5.859-863.2001

Hsieh KS, Lai TJ, Hwang YT, Lin MW, Weng KP, Chiu YT, Ho TY, Chen CS, Shiue YL, Hsiao M, Tsai SF, and Ger LP. 2011. IL-10 promoter genetic polymorphisms and risk of Kawasaki disease in Taiwan. Dis Markers 30:51-59. 10.3233/dma-2011-0765

Huang WC, Huang LM, Chang IS, Chang LY, Chiang BL, Chen PJ, Wu MH, Lue HC, and Lee CY. 2009. Epidemiologic features of Kawasaki disease in Taiwan, 2003-2006. Pediatrics 123:e401-405. 10.1542/peds.2008-2187

Jeng MJ, Lee YS, Tsao PC, Yang CF, Luo YC, and Soong WJ. 2014. A 10-year populationbased nationwide descriptive analysis of pediatric emergency care. BMC Pediatrics 14:100. 10.1186/1471-2431-14-100

Keller MA, and Stiehm ER. 2000. Passive Immunity in Prevention and Treatment of Infectious Diseases. Clinical Microbiology Reviews 13:602-614.

Kimura J, Takada H, Nomura A, Ohno T, Mizuno Y, Saito M, Kusuhara K, and Hara T. 2004. Th1 and Th2 cytokine production is suppressed at the level of transcriptional regulation in Kawasaki disease. Clin Exp Immunol 137:444-449. 10.1111/j.1365-2249.2004.02506.x

Lee SB, Kim YH, Hyun MC, Kim YH, Kim HS, and Lee YH. 2015. T-Helper Cytokine Profiles in Patients with Kawasaki Disease. Korean Circ J 45:516-521. $10.4070 / \mathrm{kcj} .2015 .45 .6 .516$

Leuridan E, Hens N, Hutse V, Ieven M, Aerts M, and Van Damme P. 2010. Early waning of maternal measles antibodies in era of measles elimination: longitudinal study. BMJ 340. 10.1136/bmj.c1626

Lin MC, Lai MS, Jan SL, and Fu YC. 2015. Epidemiologic features of Kawasaki disease in acute stages in Taiwan, 1997-2010: effect of different case definitions in claims data analysis. $J$ Chin Med Assoc 78:121-126. 10.1016/j.jcma.2014.03.009.

Liu JM, Hsu RJ, Chang FW, Yeh CL, Huang CF, Chang ST, Chiu NC, Chang HY, Chi H, and Lin CY. 2017. Increase the risk of intellectual disability in children with scabies: A 
415

416

417

418

419

420

421

422

423

424

425

426

427

428

429

430

431

432

433

434

435

436

437

438

439

440

441

442

443

444

445

446

447

448

449

nationwide population-based cohort study. Medicine 96:e7108.

10.1097/MD.0000000000007108

Malik S, Giacoia GP, and West K. 1991. The use of intravenous immunoglobulin (IVIG) to prevent infections in bronchopulmonary dysplasia: report of a pilot study. $J$ Perinatol 11:239-244.

Matsubara T, Ichiyama T, and Furukawa S. 2005. Immunological profile of peripheral blood lymphocytes and monocytes/macrophages in Kawasaki disease. Clin Exp Immunol 141:381-387. 10.1111/j.1365-2249.2005.02821.x

Milani C, Dalia SM, and Colvin GA. 2009. Thromboembolic complications of intravenous immunoglobulin (IVIG) in an immunocompromised patient with Chronic Lymphocytic Leukemia: a case report. Cases Journal 2:9078-9078. 10.1186/1757-1626-2-9078

Mouthon L, and Lortholary O. 2003. Intravenous immunoglobulins in infectious diseases: where do we stand? Clinical Microbiology and Infection 9:333-338. http://dx.doi.org/10.1046/j.1469-0691.2003.00694.x

Newburger JW, Takahashi M, and Burns JC. 2016. Kawasaki Disease. J Am Coll Cardiol 67:1738-1749. 10.1016/j.jacc.2015.12.073

Newburger JW, Takahashi M, Gerber MA, Gewitz MH, Tani LY, Burns JC, Shulman ST, Bolger AF, Ferrieri P, Baltimore RS, Wilson WR, Baddour LM, Levison ME, Pallasch TJ, Falace DA, and Taubert KA. 2004. Diagnosis, treatment, and long-term management of Kawasaki disease: a statement for health professionals from the Committee on Rheumatic Fever, Endocarditis and Kawasaki Disease, Council on Cardiovascular Disease in the Young, American Heart Association. Circulation 110:2747-2771. 10.1161/01.cir.0000145143.19711.78

Ohlsson A, and Lacy JB. 2013. Intravenous immunoglobulin for preventing infection in preterm and/or low birth weight infants. Cochrane Database Syst Rev:Cd000361. 10.1002/14651858.CD000361.pub3

Orange JS, Grossman WJ, Navickis RJ, and Wilkes MM. 2010. Impact of trough IgG on pneumonia incidence in primary immunodeficiency: A meta-analysis of clinical studies. Clin Immunol 137:21-30. 10.1016/j.clim.2010.06.012

Perez EE, Orange JS, Bonilla F, Chinen J, Chinn IK, Dorsey M, El-Gamal Y, Harville TO, Hossny E, Mazer B, Nelson R, Secord E, Jordan SC, Stiehm ER, Vo AA, and Ballow M. 2017. Update on the use of immunoglobulin in human disease: A review of evidence. $J$ Allergy Clin Immunol 139:S1-s46. 10.1016/j.jaci.2016.09.023

Siberil S, Elluru S, Negi VS, Ephrem A, Misra N, Delignat S, Bayary J, Lacroix-Desmazes S, Kazatchkine MD, and Kaveri SV. 2007. Intravenous immunoglobulin in autoimmune and 
450 inflammatory diseases: more than mere transfer of antibodies. Transfus Apher Sci $451 \quad 37: 103-107.10 .1016 /$ j.transci.2007.01.012

452 Thompson DA, Makary MA, Dorman T, Pronovost PJ. 2006. Clinical and economic outcomes of 453 hospital acquired pneumonia in intra-abdominal surgery patients. Ann Surg 243(4):547-

454 552. 10.1097/01.sla.0000207097.38963.3b

455 Wang CL, Wu YT, Liu CA, Kuo HC, and Yang KD. 2005. Kawasaki disease: infection, 456 immunity and genetics. Pediatr Infect Dis $J$ 24:998-1004.

457 Wei CC, Lin CL, Kao CH, Liao YH, Shen TC, Tsai JD, Chang YJ, and Li TC. 2014. Increased 458 risk of Kawasaki disease in children with common allergic diseases. Ann Epidemiol $45924: 340-343.10 .1016 /$ j.annepidem.2014.02.003

460 Weng KP, Wei JC, Hung YM, Huang SH, Chien KJ, Lin CC, Huang SM, Lin CL, and Cheng 461 MF. 2017. Enterovirus Infection and Subsequent Risk of Kawasaki Disease: A 462 Population-Based Cohort Study. Pediatr Infect Dis J. 10.1097/inf.0000000000001748 463 Wong PH, and White KM. 2016. Impact of Immunoglobulin Therapy in Pediatric Disease: a $464 \quad$ Review of Immune Mechanisms. Clin Rev Allergy Immunol 51:303-314. $465 \quad 10.1007 / \mathrm{s} 12016-015-8499-2$

466 Ye Q, Gong FQ, Shang SQ, and Hu J. 2016. Intravenous immunoglobulin treatment 467 responsiveness depends on the degree of CD8+ T cell activation in Kawasaki disease. $468 \quad$ Clin Immunol 171:25-31 10.1016/j.clim.2016.08.012

469 470 
Figure 1

The flow chart of enrollment of study participants. 
1996-2013 Patients with Kawasaki Disease from NHIRD $(\mathrm{N}=17,580)$

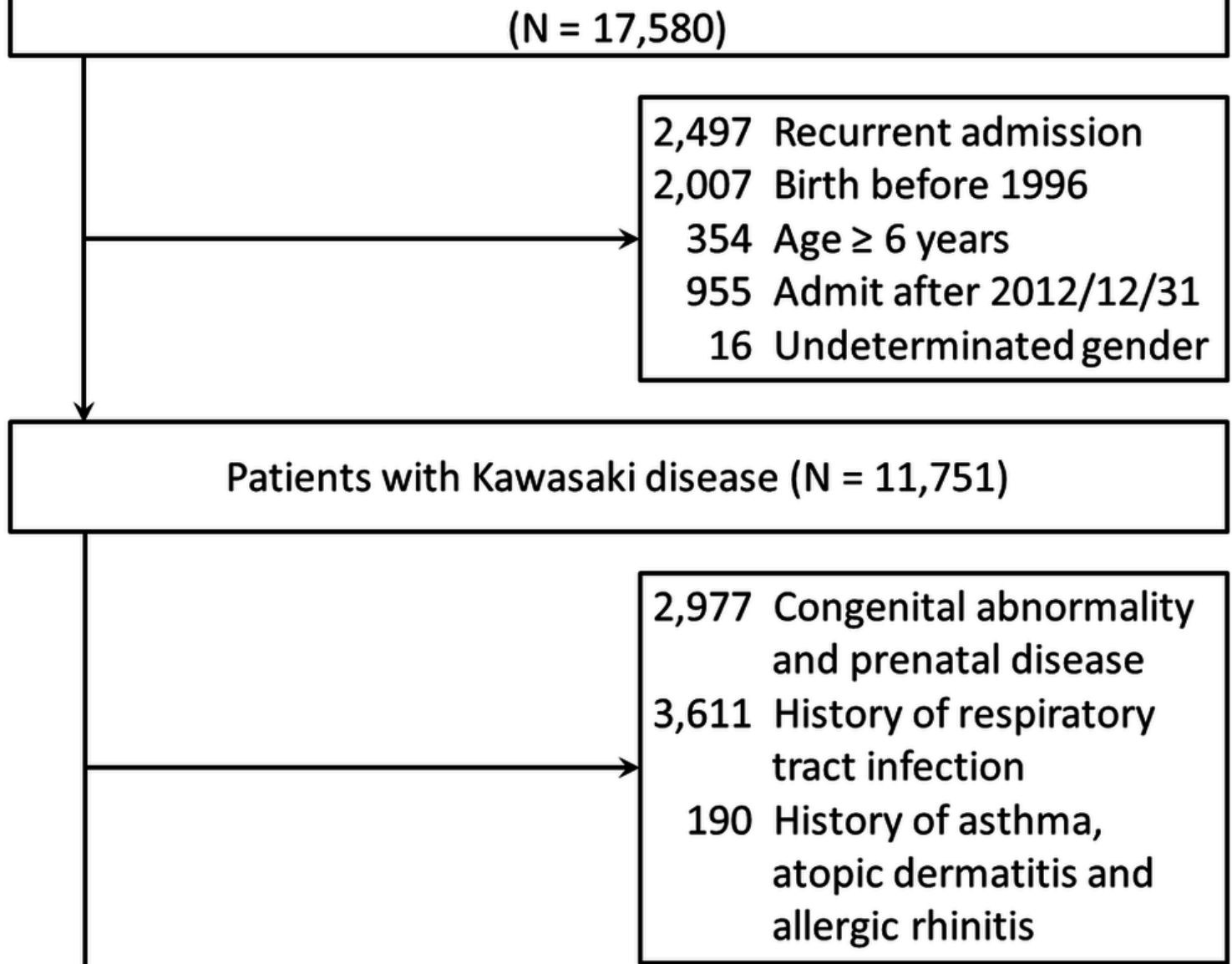

100,000 Randomized select patients born after 1996/01/01 from general population

39,486 Age $\geq 6$

1 Un-determinated gender

34 with Kawasaki disease 17,078 with admission history

Included patients with KD

$(N=4,973)$
General population

$(\mathrm{N}=43,401)$

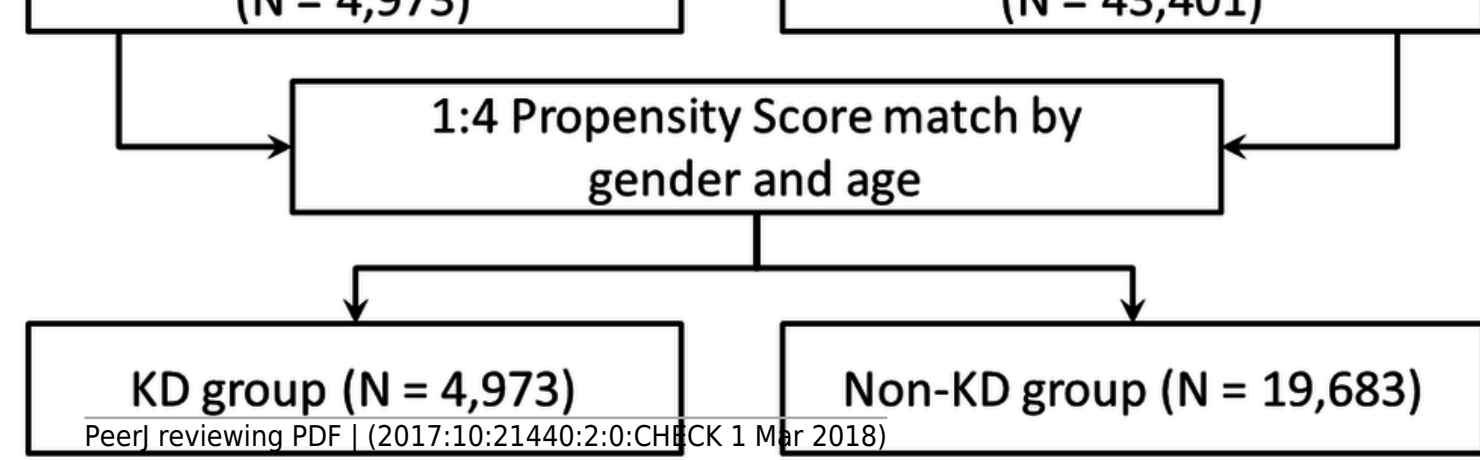


Figure 2

The Kaplan-Meier curve showed the accumulative incidences of all respiratory tract infection-related hospitalizations between KD cohort and control cohort by time ( $p$ $<0.001)$.

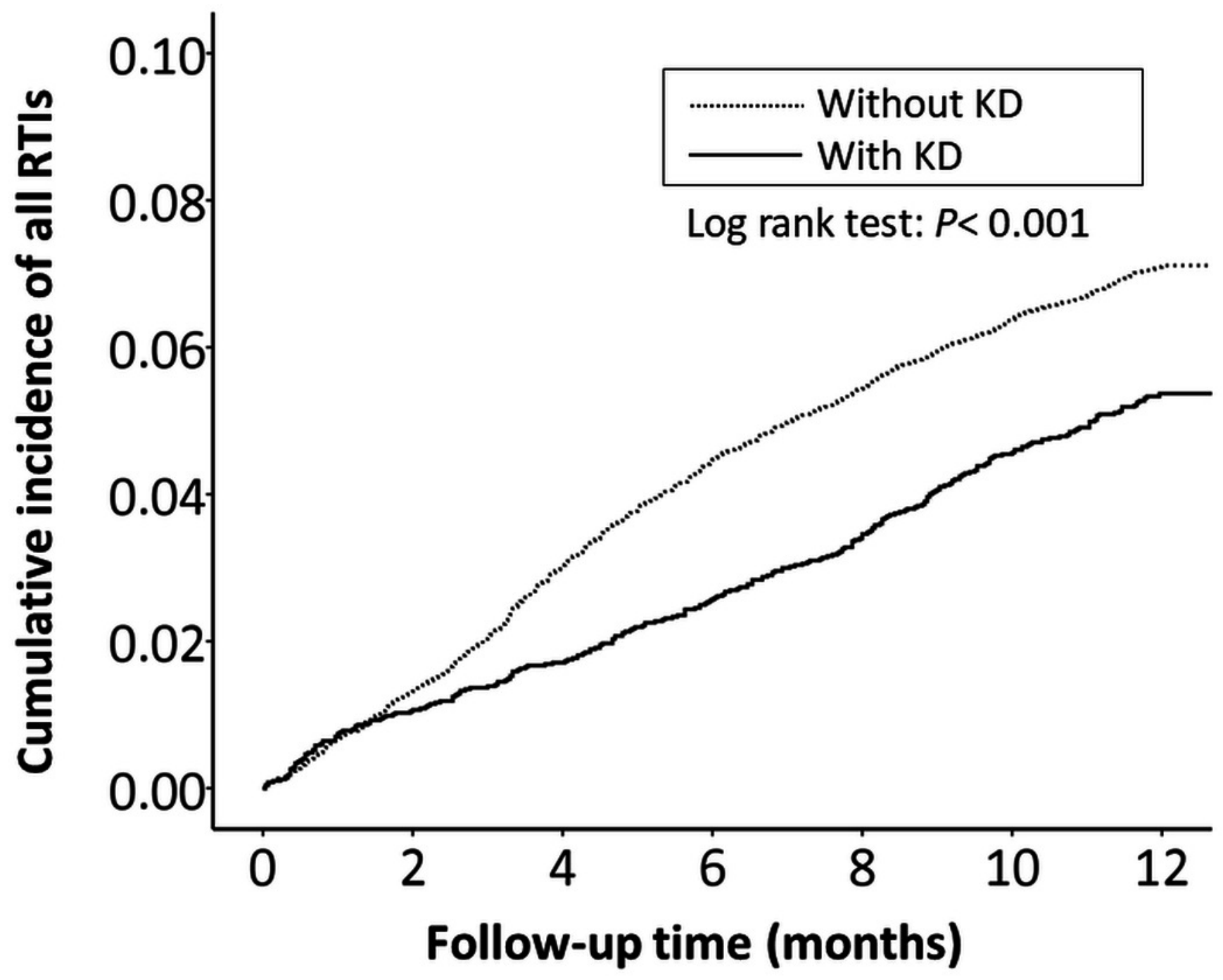


Figure 3

The Kaplan-Meier curve showed the accumulative incidences of pneumonia-related hospitalizations between KD cohort and control cohort by time $(p=0.01)$.

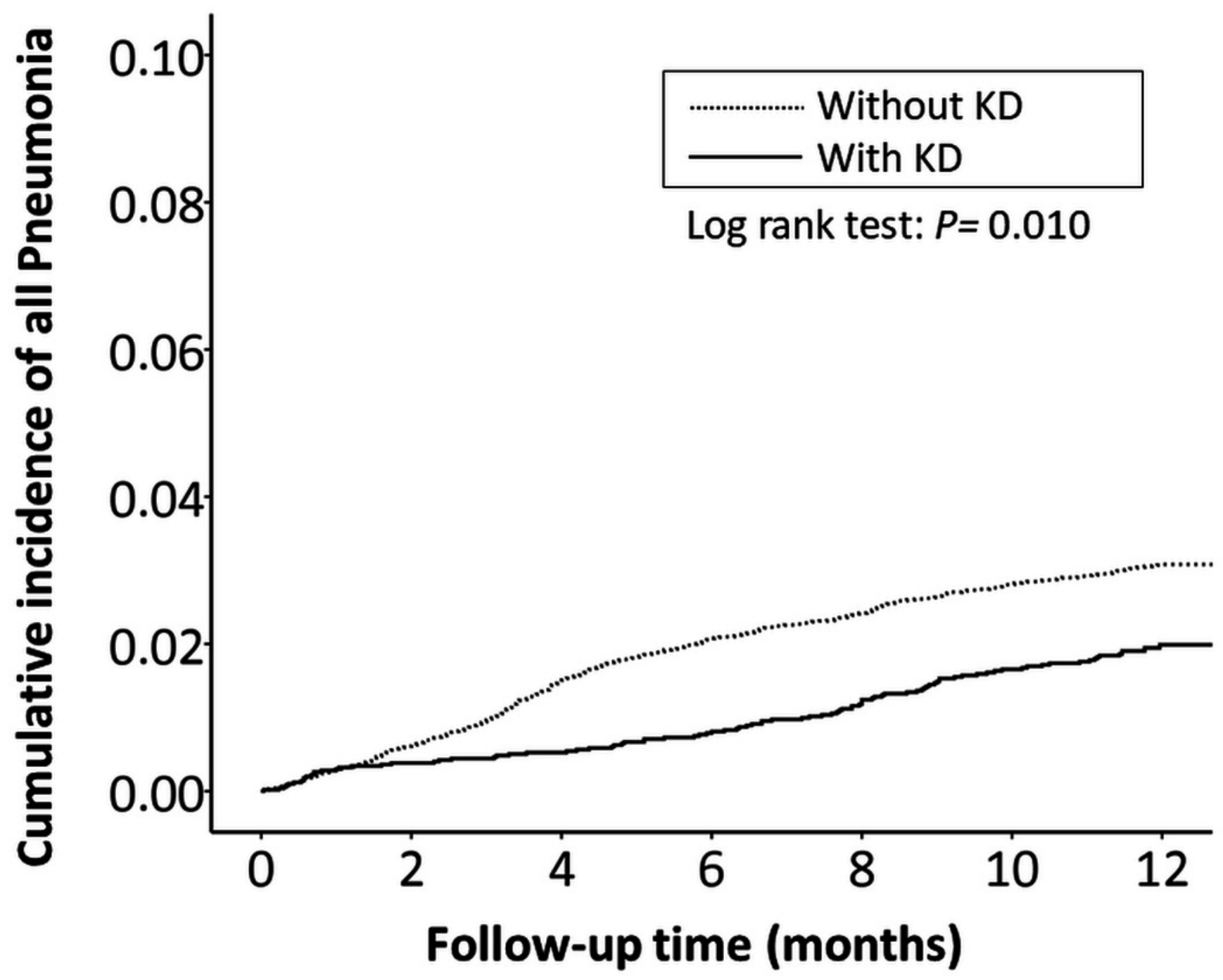


Figure 4

The Kaplan-Meier curve showed the accumulative incidences of AOM-related hospitalizations between KD cohort and control cohort by time $(p=0.01)$.

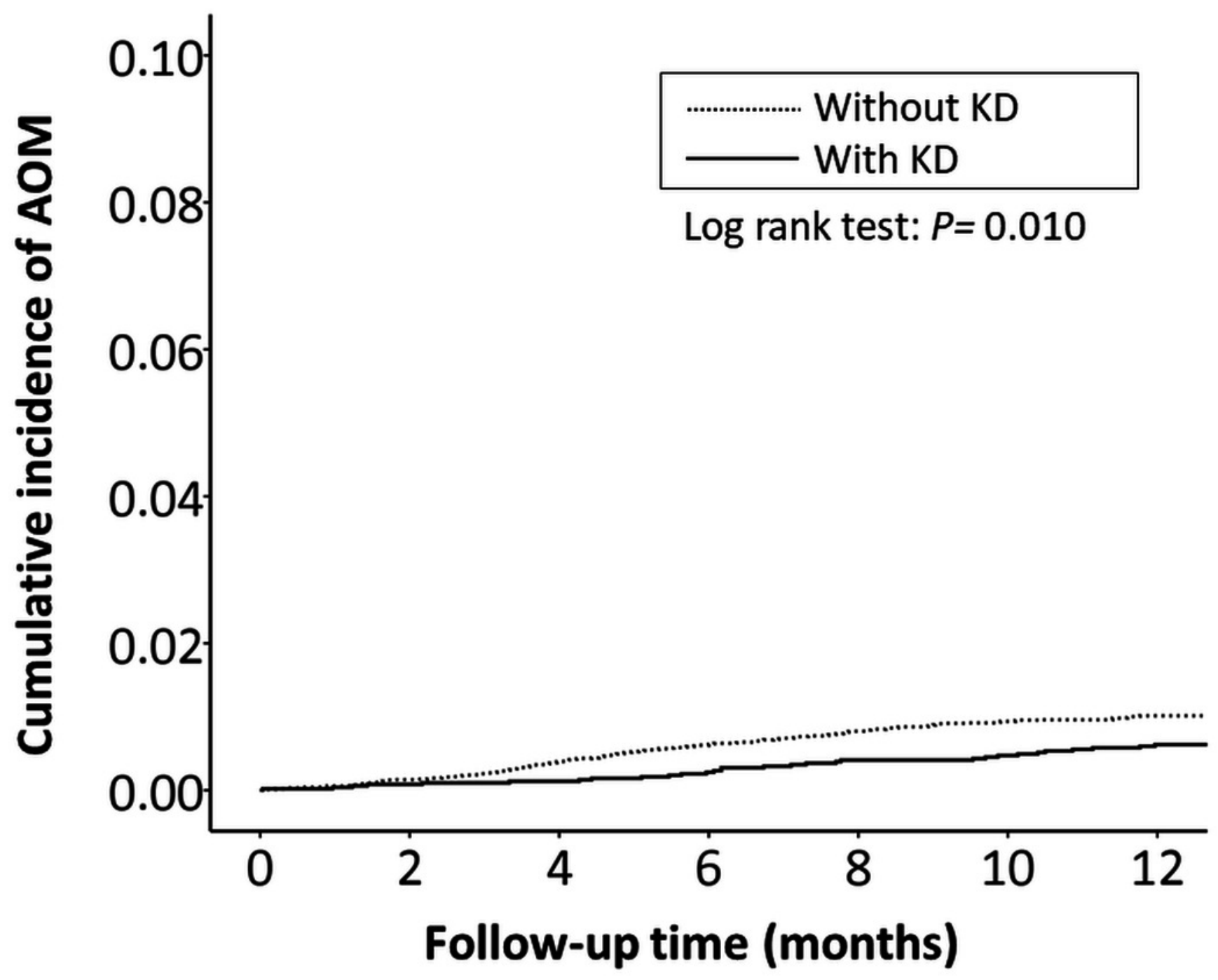


Figure 5

The Kaplan-Meier curve showed the accumulative incidences of bronchiolitis-related hospitalizations between KD cohort and control cohort by time $(p=0.033)$.

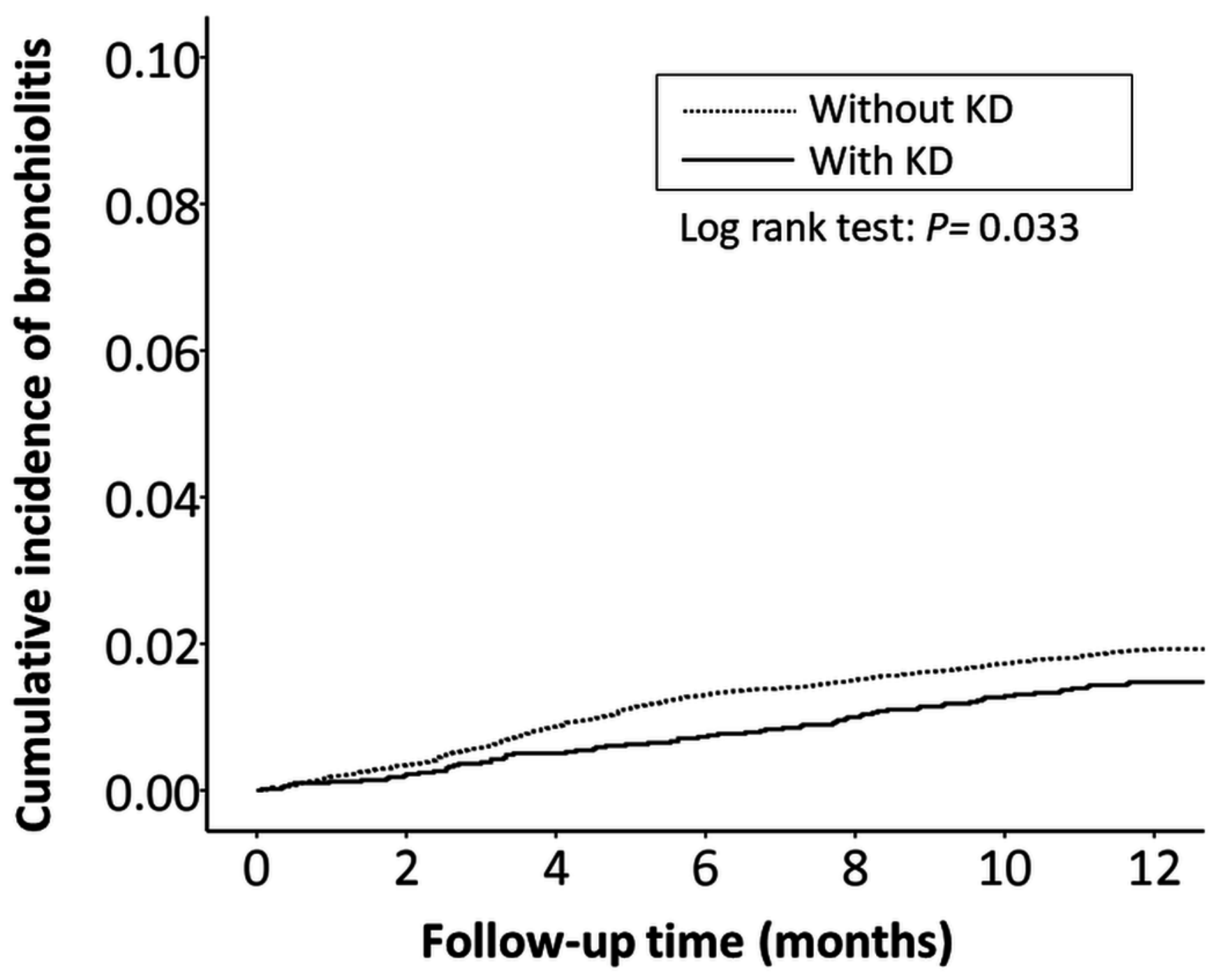




\section{Table 1 (on next page)}

Demographics between Kawasaki disease group and non-Kawasaki disease group 
Table 1. Demographics between Kawasaki disease group and nonKawasaki disease group

\begin{tabular}{llllll}
\hline Characteristics & \multicolumn{2}{c}{ KD $(\mathrm{N}=4973)$} & \multicolumn{2}{c}{ Non-KD $(\mathrm{N}=19683)$} & P value \\
& No. & $\%$ & No. & $\%$ & \\
\hline Age group & & & & & 0.914 \\
0-2 y & 3670 & 73.80 & 14542 & 73.88 & \\
2-6 y & 1303 & 26.20 & 5141 & 26.12 & \\
Mean+-SD & $1.57+-1.23$ & & $1.50+-1.07$ & & 0.339 \\
Gender & & & & & 0.583 \\
Boy & 2951 & 59.34 & 11595 & 58.91 & \\
Girl & 2022 & 40.66 & 8088 & 41.09 & \\
\hline
\end{tabular}




\section{Table 2 (on next page)}

Incidence and aHR of respiratory tract infection-related hospitalization stratified by sex, age, between KD and non-KD cohorts. 
Table 2. Incidence and aHR of respiratory tract infection-related hospitalization stratified by sex, age, between KD and nonKD cohorts.

\begin{tabular}{|c|c|c|c|c|c|c|c|c|}
\hline \multirow[b]{2}{*}{ Variables } & \multicolumn{3}{|c|}{ KD } & \multicolumn{3}{|c|}{ Non-KD } & \multicolumn{2}{|c|}{ Compared with non-KD } \\
\hline & Event & Person months & Rate & Event & Person month & Rate & IRR $(95 \% \mathrm{Cl})$ & $\operatorname{aHR}(95 \% \mathrm{Cl})$ \\
\hline \multirow{2}{*}{\multicolumn{9}{|c|}{$\begin{array}{l}\text { Overall infection } \\
\text { rate }\end{array}$}} \\
\hline & & & & & & & & \\
\hline All respiratory & 267 & 1597 & 16.72 & 1400 & 7298 & 19.18 & $0.87(0.76-0.99)^{*}$ & $0.75(0.66-0.85) \dagger$ \\
\hline Pneumonia & 97 & 614 & 15.79 & 595 & 2899 & 20.52 & $0.77(0.61-0.96)^{*}$ & $0.64(0.52-0.79) \dagger$ \\
\hline AOM & 30 & 201 & 14.93 & 193 & 1021 & 18.89 & $0.79(0.52-1.17)$ & $0.61(0.42-0.90)^{*}$ \\
\hline Bronchiolitis & 72 & 424 & 16.99 & 370 & 1840 & 20.10 & 0.85 (0.65-1.09) & $0.77(0.60-0.99)^{*}$ \\
\hline Tonsillitis & 51 & 294 & 17.35 & 192 & 1160 & 16.55 & $1.05(0.75-1.43)$ & $1.03(0.76-1.40)$ \\
\hline \multicolumn{9}{|l|}{ Sex } \\
\hline Boy & 176 & 1060 & 16.60 & 917 & 4723 & 19.41 & $0.85(0.72-1.01)$ & $0.75(0.64-0.88) \dagger$ \\
\hline Girl & 91 & 536 & 16.97 & 483 & 2574 & 18.76 & $0.90(0.71-1.13)$ & $0.75(0.60-0.94)^{*}$ \\
\hline \multicolumn{9}{|l|}{ Age (y) } \\
\hline $0-2$ & 209 & 1296 & 16.13 & 1185 & 6127 & 19.34 & $0.83(0.72-0.97)^{*}$ & $0.69(0.59-0.80) \dagger$ \\
\hline $2-6$ & 58 & 301 & 19.25 & 215 & 1171 & 18.36 & $1.05(0.77-1.41)$ & $1.07(0.80-1.43)$ \\
\hline
\end{tabular}

${ }^{*} p<0.05, \dagger p<0.001$; IRR: incidence rate ratio; aHR: multiple analysis including sex, age; Rate: incidence rate (per 1000 person months) 


\section{Table 3(on next page)}

Incidence and aHR of respiratory tract infection-related admission between KD and nonKD cohorts within the 1-year follow up 
Table 3. Incidence and aHR of respiratory tract infection-related admission between KD and non-KD cohorts within the 1-year follow up

\begin{tabular}{|c|c|c|c|c|c|c|c|c|}
\hline \multirow[b]{2}{*}{ Variables } & \multicolumn{2}{|r|}{ KD } & \multirow[b]{2}{*}{ Rate } & \multicolumn{2}{|c|}{ Non-KD } & \multicolumn{3}{|c|}{ Compared with non-KD } \\
\hline & Event & Person months & & Event & Person month & Rate & $\operatorname{IRR}(95 \% \mathrm{Cl})$ & $\operatorname{aHR}(95 \% \mathrm{Cl})$ \\
\hline \multicolumn{9}{|c|}{ Follow-up time, (m) } \\
\hline $0-3$ & 69 & 83 & 83.50 & 405 & 636 & 80.64 & $1.31(1.00-1.70)^{*}$ & $0.68(0.53-0.88)^{*}$ \\
\hline $3-6$ & 59 & 268 & 21.98 & 475 & 2079 & 22.65 & $0.96(0.72-1.26)$ & $0.49(0.37-0.64) \dagger$ \\
\hline $6-9$ & 74 & 568 & 13.02 & 290 & 2175 & 13.34 & $0.98(0.75-1.26)$ & $0.99(0.77-1.28)$ \\
\hline $9-12$ & 65 & 677 & 9.60 & 230 & 2408 & 9.55 & $1.00(0.75-1.33)$ & $1.10(0.84-1.45)$ \\
\hline
\end{tabular}

$* p<0.05, \dagger p<0.001$; IRR: incidence rate ratio; aHR: multiple analysis including sex, age; Rate: incidence rate (per 1000 person months) 